\title{
LOWER CARDIAC VAGAL TONE IN NON- OBESE HEALTHY MEN WITH UNFAVORABLE ANTHROPOMETRIC CHARACTERISTICS
}

\author{
Plínio S. Ramos, ${ }^{\text {I Claudio Gil S. Araújo }}$ I, II
}

doi: 10.1590/S1807-59322010000100008

Ramos PS, Araújo CGS. Lower cardiac vagal tone in non-obese healthy men with unfavorable anthropometric characteristics. Clinics. 2010;65(1):45-51.

OBJECTIVES: to determine if there are differences in cardiac vagal tone values in non-obese healthy, adult men with and without unfavorable anthropometric characteristics.

INTRODUCTION: It is well established that obesity reduces cardiac vagal tone. However, it remains unknown if decreases in cardiac vagal tone can be observed early in non-obese healthy, adult men presenting unfavorable anthropometric characteristics.

METHODS: Among 1688 individuals assessed between 2004 and 2008, we selected 118 non-obese (BMI $<30 \mathrm{~kg} / \mathrm{m}^{2}$ ), healthy men (no known disease conditions or regular use of relevant medications), aged between 20 and 77 years old (42 \pm 12 -years-old). Their evaluation included clinical examination, anthropometric assessment (body height and weight, sum of six skinfolds, waist circumference and somatotype), a 4-second exercise test to estimate cardiac vagal tone and a maximal cardiopulmonary exercise test to exclude individuals with myocardial ischemia. The same physician performed all procedures.

RESULTS: A lower cardiac vagal tone was found for the individuals in the higher quintiles - unfavorable anthropometric characteristics - of BMI ( $\mathrm{p}=0.005)$, sum of six skinfolds $(\mathrm{p}=0.037)$ and waist circumference $(\mathrm{p}<0.001)$. In addition, the more endomorphic individuals also presented a lower cardiac vagal tone $(\mathrm{p}=0.023)$, while an ectomorphic build was related to higher cardiac vagal tone values as estimated by the 4 -second exercise test $(\mathrm{r}=0.23 ; \mathrm{p}=0.017)$.

CONCLUSIONS: Non-obese and healthy adult men with unfavorable anthropometric characteristics tend to present lower cardiac vagal tone levels. Early identification of this trend by simple protocols that are non-invasive and risk-free, using select anthropometric characteristics, may be clinically useful in a global strategy to prevent cardiovascular disease.

KEYWORDS: Waist circumference; Anthropometric; Body mass index; Somatotype; Autonomic nervous system.

\section{INTRODUCTION}

More than a billion people around the world are overweight, and approximately 300 million are obese. ${ }^{1}$ Obesity is considered an important independent risk factor for the development of cardiovascular disease (CVD) $)^{2-4}$ and mortality. ${ }^{5}$ Several pathophysiological mechanisms may explain the strong association between obesity and the

I Programa de Pós-Graduação em Educação Física, Universidade Gama Filho - Rio de Janeiro/RJ, Brasil.

II Clínica de Medicina do Exercício (CLINIMEX) - Rio de Janeiro/RJ,Brasil. Email: cgaraujo@iis.com.br

Tel: 55212256.7183

Received for publication on August 26, 2009

Accepted for publication on October 19, 2009 risk of developing CVD, including autonomic dysfunction, which, due to a reduced cardiac vagal tone (CVT), ${ }^{6}$ can alone be an important risk factor for $\mathrm{CVD}^{7,8}$ and mortality. ${ }^{9}$

Studies have shown that obesity, evaluated by simple indicators such as body mass index (BMI) (weight/ height ${ }^{2}$ ), fat regional indicators or total body fat (i.e., waist circumference and sum of skinfolds, respectively), is associated with a reduced CVT. ${ }^{10-14}$ For example, Freeman et al. ${ }^{14}$ suggested that CVT tends to be diminished in individuals with higher BMI. A study of obese subjects by Chen et al. ${ }^{13}$ demonstrated a better association between CVT and indicators of central obesity (i.e., waist circumference) as compared to total indicators of obesity, such as BMI. 
In theory, it is possible that some degree of CVT reduction can anticipate the appearance of clinical signs and symptoms of certain diseases. As such, CVT may function as a pre-clinical sign that can be potentially useful for clinical decision making. Although current evidence supports the hypothesis that CVT varies inversely with indicators of obesity, ${ }^{15,16}$ an analysis of CVT in healthy adult men who are not obese but are within the upper limits of normality for some selected and unfavorable anthropometric characteristics (UAC), such as being overweight, having an increased waist circumference and presenting a predominantly endomorphic somatotype, has not yet been carried out. Therefore, our aim was to determine if there are differences in CVT values in nonobese healthy, adult men (BMI $<30 \mathrm{~kg} / \mathrm{m}^{2}$ ) with and without UAC and to relate CVT to select anthropometric measurements.

\section{METHODS}

Data from 1688 consecutive detailed medical-functional evaluations between 2004 and 2008 were reviewed, and 118 evaluations were found to meet all of the following inclusion criteria: a) male gender; b) aged 20 to 77 -yearsold; c) healthy (absence of known clinical relevant diseases and no regular use of medications, except for vitamins; d) BMI $<30 \mathrm{~kg} / \mathrm{m}^{2}$; and e) having completed a truly maximal cardiopulmonary exercise test. Evaluation of the subjects included clinical examination, anthropometric assessment (body height and weight, sum of six skinfolds, waist circumference and somatotype), 4-second exercise test $(4 \mathrm{sET})^{17}$ (for CVT estimation) and a maximal cardiopulmonary exercise test (CPET).$^{18}$ All procedures were carried out by the same physician. Evaluations were performed at the request of the individuals, most commonly by referral from their primary care physicians. These apparently healthy individuals seek our advice, in most cases, to assess their current physical condition and to get subsides in order to increase regular exercise and/or in adopting a healthier lifestyle. These individuals have a high possibility of being truly healthy, not only due to the absence of known diseases as assessed by their physicians but also by denying regular use of relevant medications. Furthermore, no pathological findings were detected after a detailed clinical evaluation, which included a maximal CPET, which was performed to exclude myocardial ischemia and to identify appropriate heart rate target zones for aerobic exercise prescription. Before the procedures, all subjects read and signed a specific informed consent form previously approved by the institutional research committee.

\section{Anthropometric Measurements}

Body weight was measured with an electronic scale to the nearest $0.01 \mathrm{~kg}$, and height was measured to the nearest $0.1 \mathrm{~cm}$ using a fixed stadiometer. We obtained skinfolds measurements at six body sites (triceps, subscapular, suprailiac, abdomen, thigh and medial calf) by a caliper (Skyndex II, USA) read to the nearest 0.1 $\mathrm{mm}$. Circumference measurements, to the nearest 0.1 $\mathrm{cm}$, of waist, calf, relaxed and the flexed/contracted right arm, were taken with a metallic and flexible tape (Gullick, USA). Humeral and femoral bone diameters were obtained by using an adapted sliding caliper (Mitutoyo, Japan) and were measured to the nearest $0.01 \mathrm{~mm}$. As previously stated, all anthropometric measurements were obtained by a single and experienced physician following ISAK standards. ${ }^{19}$

\section{Anthropometric characteristics (AC)}

\section{Total}

The total anthropometric characteristics were evaluated in three ways. The BMI was obtained from the ratio of body mass $(\mathrm{kg})$ to squared height $(\mathrm{m})$. Total fat was estimated by adding the values of six skinfolds from the triceps, subscapular, suprailiac, abdomen, thigh and the medial calf sites in $\mathrm{mm}$. We determine the Heath-Carter somatotype anthropometric method ${ }^{20,21}$ allowing a more comprehensive body composition analysis by the evaluation of three components: a) the endomorphy, which represents relative fat, b) the mesomorphy, which represents relative skeletal and muscular development and c) the ectomorphy, which reflects the relative linearity $=[2.42 \times(($ height $(\mathrm{cm}) / 2.54) /$ (weight $(\mathrm{kg}) / 0.4536) 1 / 3)-28.58] .{ }^{22}$

\section{Regional}

Regional AC was evaluated by two approaches: a) peripheral central Index (PCI), obtained from the ratio of the sum of central and peripheral skinfolds, where the central sum is obtained by adding abdominal, suprailiac and subscapular skinfold values, while the peripheral sum is achieved by adding triceps, thigh and medial calf skinfold values; and b) waist circumference, measured at the umbilical scar level and parallel to the floor.

\section{UAC}

For the purpose of our study, the results at upper extreme (lower extreme for ectomorphy) of distribution for each one of anthropometric characteristics above described were labeled as UAC. Further details are provided under statistical analysis subsection. 


\section{Assessment of cardiac vagal tone (CVT):}

To assess the CVT, the rapid initial heart rate transient (i.e., rest-exercise transition), represented by the cardiac vagal index (CVI), was determined by the 4sET protocol. Briefly, the 4sET consists in unloaded pedaling as fast as possible on a cycle ergometer from the $5^{\text {th }}$ to the $8^{\text {th }}$ second of a 12-s maximal inspiratory apnea. For testing, the subject remains seated on the cycle ergometer and, after the heart rate (HR) stabilizes, four verbal commands guide the actions that are to be sequentially performed, at 4-s intervals, as follows: 1) fast maximal inspiration, primarily through the mouth; 2) pedaling as fast as possible; 3 ) sudden cessation of pedaling; and 4) expiration. During the 4sET, a digital ECG was recorded with 10-ms resolution. The CVI was calculated from the ratio between two ECG specific R-R intervals (with 10-ms resolution) were identified and measured: the longest R-R interval (RRB) (i.e. either the interval obtained immediately before the onset of exercise or the first one after the onset of exercise) and the shortest R-R interval (RRC) during the 4-s exercise (generally, the last one). The CVI was calculated from the ratio between the RRB and RRC interval. ${ }^{17,18}$ Two 4 sET maneuvers were performed and the highest ratio between the RRB and RRC intervals which are representative of the CVI, a dimensionless measurement, used for further analysis.

\section{Maximal cardiopulmonary exercise test (CPET):}

Following the 4sET, a CPET was performed - leg cycle ergometer in $96 \%$ of the tests - with direct collection and analysis of expired gases (VO2000, Medgraphics, USA) according to an individualized ramp protocol, aimed to last between 8 and 12 minutes. ${ }^{23,24}$ The subjects were verbally encouraged to exercise until they reached volitional fatigue (i.e., exhaustion), regardless of the maximal heart rate achieved or any other ventilatory criteria. One lead ECG (CC5 or CM5) was continuously monitored from rest until at least 5 min post-exercise. ${ }^{18,25}$ Auscultatory blood pressure measurements were also obtained at each minute during exercise and recovery.

\section{Statistical analysis}

Two different analysis were conducted. In the first approach, the samples were divided into quintiles for ACs, as follows: a) BMI; b) total sum of six skinfolds; c) PCI; d) waist circumference; and e) somatotype components (endomorph, mesomorph and ectomorph). After that, paired one-tailed t-tests were used to compare the extreme quintiles - lower and higher UAC values. In the second approach, the association between each one of the ACs and the CVI was determined by Pearson product-moment correlation. Additionally, a one-way ANOVA was carried out for comparing age of subjects of the five quintiles for each one of the ACs analyzed. A value of $5 \%$ was considered significant. All calculations were performed using SPSS software (version 17, SPSS, Chicago).

\section{RESULTS}

Demographic descriptive statistical data are presented in Table 1 and physiological testing data are shown in Table 2. Table 3 displays the quintile cut-off values for each one of the selected AC. No significant differences were found for age among the quintiles for all ACs variables assessed.

Table 1- Demographic characteristics of the participants (n $=118$ )

\begin{tabular}{lcc}
\hline Variable $(\mathrm{n}=118)$ & Mean $\pm \mathrm{SD}$ & Minimum - Maximum \\
\hline Age $(\mathrm{years})$ & $42 \pm 12$ & $20-77$ \\
Height $(\mathrm{m})$ & $1.76 \pm 0.06$ & $1.58-1.94$ \\
Weight $(\mathrm{kg})$ & $79.5 \pm 8.4$ & $58.5-99.7$ \\
BMI $\left(\mathrm{kg} / \mathrm{m}^{2}\right)$ & $25.6 \pm 2.2$ & $20.7-29.8$ \\
$\sum$ Total $(\mathrm{mm})$ & $87.2 \pm 26.1$ & $32.2-156.2$ \\
Waist circumference $(\mathrm{cm})$ & $90.2 \pm 7$ & $71.3-113.0$ \\
PCI & $1.8 \pm 0.5$ & $0.64-3.76$ \\
Endomorph & $4.1 \pm 1.25$ & $1.33-7.24$ \\
Mesomorph & $5.71 \pm 0.93$ & $3.82-7.91$ \\
Ectomorph & $1.46 \pm 1.01$ & $-1.09-3.70$ \\
\hline
\end{tabular}

BMI, Body mass index; PCI, Peripheral central index; $\sum$ total, Total sum of skinfolds

Table 2 - 4sET and maximal cardiopulmonary exercise testing (CPET) major findings

\begin{tabular}{lcc}
\hline Variable $(\mathrm{n}=118)$ & Mean $\pm \mathrm{DP}$ & Minimum - Maximum \\
\hline Cardiac Vagal Index & $1.63 \pm 0.33$ & $1.11-2.67$ \\
SBPrest $(\mathrm{mmHg})$ & $127 \pm 10.8$ & $106-159$ \\
DBPrest $(\mathrm{mmHg})$ & $74 \pm 8$ & $53-102$ \\
HRrest $(\mathrm{bpm})$ & $60 \pm 11$ & $39-96$ \\
HRmax $(\mathrm{bpm})$ & $179 \pm 15$ & $133-211$ \\
\% HRmax & $98 \pm 7$ & $78-118$ \\
HRR (bpm) & $41 \pm 11$ & $16-65$ \\
$\%$ VO2max & $125 \pm 31.2$ & $42-203$ \\
METs & $13.2 \pm 3.5$ & $4.7-21.1$ \\
\hline
\end{tabular}

SBPrest, resting sistolic blood pressure; DBPrest, resting diastolic blood pressure; HRrest, resting heart rate; HRmax, maximal heart rate during cardipulmonary exercise testing; \%HRmax, \% of predicted HRmax achieved; HRR, heart rate recovery (i.e. HRmax minus HR at first min of recovery); METs, Metabolic equivalent. 
Table 3 - Quintiles of selected anthropometric characteristics

\begin{tabular}{|c|c|c|c|c|}
\hline \multirow[b]{2}{*}{ Variable } & \multirow[b]{2}{*}{ Quintile } & \multirow[t]{2}{*}{ cut-of points } & \multirow[b]{2}{*}{$\mathbf{n}$} & \multirow{2}{*}{$\begin{array}{l}\text { Cardiac Vagal } \\
\text { Index } \\
(\text { mean } \pm \mathrm{SEM})\end{array}$} \\
\hline & & & & \\
\hline \multirow{5}{*}{ BMI $\left(\mathbf{k g} / \mathbf{m}^{2}\right)$} & $1 \mathrm{st}$ & $\leq 23.6$ & 23 & $1.78 \pm 0.07$ \\
\hline & $2 \mathrm{nd}$ & $23.7-25.0$ & 25 & $1.60 \pm 0.06$ \\
\hline & $3 \mathrm{rd}$ & $25.1-26.2$ & 23 & $1.67 \pm 0.07$ \\
\hline & 4 th & $26.3-27.8$ & 24 & $1.59 \pm 0.07$ \\
\hline & 5 th & $\geq 27.9$ & 23 & $1.52 \pm 0.07$ \\
\hline \multirow{5}{*}{$\sum$ total $(\mathbf{m m})$} & 1 st & $\leq 68.3$ & 24 & $1.74 \pm 0.08$ \\
\hline & 2 nd & $68.4-79.2$ & 23 & $1.67 \pm 0.06$ \\
\hline & $3 \mathrm{rd}$ & $79.3-91.0$ & 23 & $1.64 \pm 0.07$ \\
\hline & 4th & $91.1-107.5$ & 24 & $1.56 \pm 0.06$ \\
\hline & 5 th & $\geq 107.6$ & 23 & $1.54 \pm 0.07$ \\
\hline \multirow{5}{*}{ PCI } & 1 st & $\leq 1.27$ & 24 & $1.58 \pm 0.07$ \\
\hline & 2 nd & $1.28-1.66$ & 24 & $1.66 \pm 0.06$ \\
\hline & $3 \mathrm{rd}$ & $1.67-1.94$ & 23 & $1.73 \pm 0.08$ \\
\hline & 4 th & $1.95-2.20$ & 24 & $1.66 \pm 0.07$ \\
\hline & 5 th & $\geq 2.21$ & 23 & $1.53 \pm 0.06$ \\
\hline \multirow{5}{*}{$\begin{array}{l}\text { Waist circum- } \\
\text { ference }(\mathrm{cm})\end{array}$} & 1 st & $\leq 84.7$ & 24 & $1.77 \pm 0.07$ \\
\hline & 2 nd & $84.8-87.7$ & 24 & $1.68 \pm 0.06$ \\
\hline & $3 \mathrm{rd}$ & $87.8-91.5$ & 23 & $1.61 \pm 0.06$ \\
\hline & 4th & $91.6-96.2$ & 25 & $1.63 \pm 0.08$ \\
\hline & 5 th & $\geq 96.3$ & 22 & $1.46 \pm 0.04$ \\
\hline \multirow{5}{*}{ Endomorphy } & 1 st & $\leq 2.90$ & 24 & $1.77 \pm 0.07$ \\
\hline & $2 \mathrm{nd}$ & $2.91-3.73$ & 24 & $1.62 \pm 0.07$ \\
\hline & $3 \mathrm{rd}$ & $3.74-4.48$ & 23 & $1.63 \pm 0.06$ \\
\hline & 4 th & $4.49-5.24$ & 24 & $1.55 \pm 0.07$ \\
\hline & 5 th & $\geq 5.25$ & 23 & $1.58 \pm 0.07$ \\
\hline \multirow{5}{*}{ Mesomorphy } & 1 st & $\leq 4.78$ & 24 & $1.60 \pm 0.06$ \\
\hline & 2 nd & $4.79-5.50$ & 24 & $1.67 \pm 0.06$ \\
\hline & $3 \mathrm{rd}$ & $5.51-5.98$ & 23 & $1.76 \pm 0.08$ \\
\hline & 4 th & $5.99-6.53$ & 24 & $1.52 \pm 0.07$ \\
\hline & 5 th & $\geq 6.54$ & 23 & $1.60 \pm 0.07$ \\
\hline \multirow{5}{*}{ Ectomorphy } & 1 st & $\leq 0.48$ & 24 & $1.55 \pm 0.07$ \\
\hline & 2 nd & $0.49-1.19$ & 24 & $1.58 \pm 0.07$ \\
\hline & $3 \mathrm{rd}$ & $1.20-1.68$ & 23 & $1.67 \pm 0.07$ \\
\hline & 4 th & $1.69-2.31$ & 24 & $1.59 \pm 0.06$ \\
\hline & 5 th & $\geq 2.32$ & 23 & $1.78 \pm 0.07$ \\
\hline
\end{tabular}

BMI: Body mass index; PCI: Peripheral central index; $\sum$ total: Total sum of skinfolds.

With respect to the regional $\mathrm{AC}$, waist circumference showed a trend similar to the total body fat indicators described above, with the first quintile of individuals showing higher CVT values as compared to those in the fifth
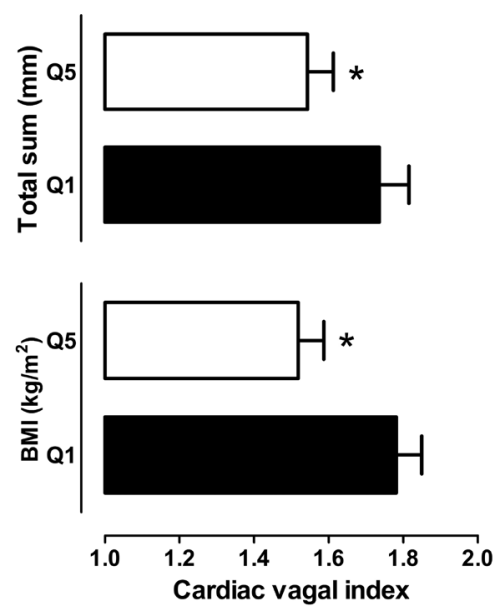

Figure 1 - Total body fat unfavorable anthropometric characteristics, * $\mathrm{p}<0.05$

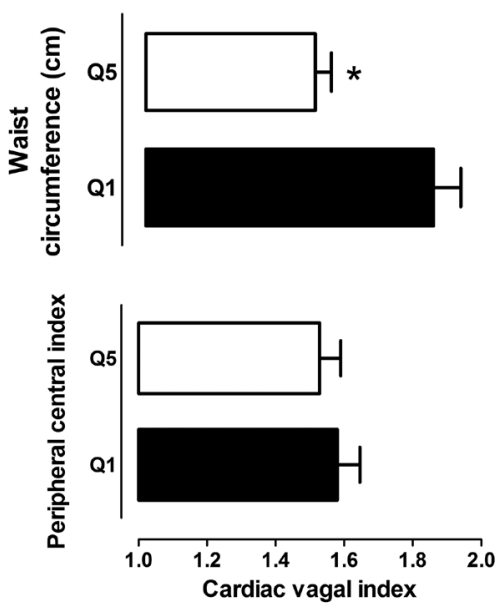

Figure 2 - Regional unfavorable anthropometric characteristics, ${ }^{*} \mathrm{p}<0.05$

quintile (1.77 vs. $1.46, \mathrm{p}<0.001$ ) (Figure 2 ). On the other hand, no differences were observed in the CVT concerning the PCI of extreme quintiles ( 1.58 vs. $1.53, \mathrm{p}=0.284$ ).

Moving to somatotype analysis, CVT differences were found for the individuals in the extreme quintiles for the endomorphy ( 1.77 vs. $1.56, \mathrm{p}=0.023)$, with the more endomorphic individuals showing lower CVI values. The more linear individuals, as represented by the fifth quintile of ectomorphy showed greater CVI values as compared with those with a more rounded body shape (1.78 vs. 1.55 , $\mathrm{p}=0.013$ ). On the other hand, evaluation of the CVT for the mesomorphy, failed to show any difference between the extreme quintiles (1.60 vs. $1.60, \mathrm{p}=1.000$ ). (Figure 3 ).

Looking for associations, we found that UAC results, except for mesomorphy, are related to CVT, represented by the CVI, with correlation coefficients (Table 4) ranging, in module, between 0.2 and $0.3(\mathrm{p}<0.05)$. Since endomorphy and ectomorphy tend to be inversely related, the $r$ values of these variables with CVT also follow the same pattern. 

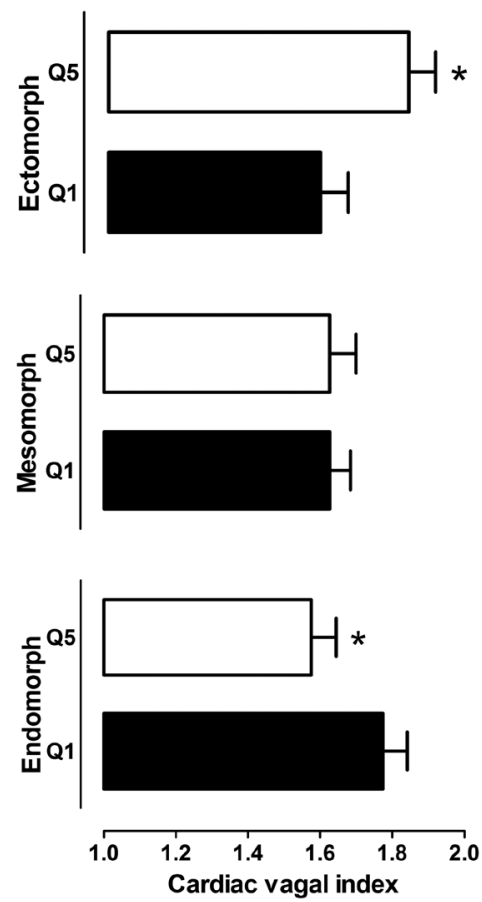

Figure 3 - Somatotype ${ }^{*} \mathrm{p}<0.05$

Table 4 - Correlation coefficients between selected anthropometric characteristics and the cardiac vagal index

\begin{tabular}{lcc}
\hline Variable & \multicolumn{2}{c}{ Cardiac Vagal Index (CVI) } \\
$\mathrm{n}=118$ & $\mathrm{r}$ & $\mathrm{p}$ \\
\hline AC total & -0.20 & 0.024 \\
$\mathrm{BMI}\left(\mathrm{kg} / \mathrm{m}^{2}\right)$ & -0.23 & 0.013 \\
$\sum$ total $(\mathrm{mm})$ & & \\
\hline AC regional & -0.30 & 0.001 \\
Waist Circumference $(\mathrm{cm})$ & -0.02 & 0.842 \\
PCI & & \\
\hline Somatotype & -0.24 & 0.011 \\
Endomorphy & 0.23 & 0.017 \\
Ectomorphy &
\end{tabular}

AC: Anthropometric characteristics; BMI: Body mass index; PCI: Peripheral central index; $\Sigma$ total: Total sum of skinfolds.

\section{DISCUSSION}

This study suggested that non-obese and healthy adult men, as considered by not having any relevant clinical diagnosis and not been in regular use of medications, but who already have UAC, present a significantly lower CVT when compared with a comparable group of individuals without these characteristics. However, it is important to emphasize that although these individuals present a reduction in CVT, they are still within the normal range of CVI values $(1,2$ to 1,9 , based on unpublished results based in a sample of more than 1,8004 sETs carried out in healthy adults in the last 20 years). Age could be a relevant intervenient variable, since most of the studies recognized a significant decrease in CVT with aging ${ }^{7}$, however, in this particular study, aging is unlikely to have interfered since there was no difference among the five quintiles for this variable. A peculiarity of this study is the rigid selection of individuals with a high possibility of being truly healthy, not only due to the absence of known diseases as assessed by their physicians but also by not reporting use of regular medical. Furthermore, no pathological findings were detected after all selected subjects have underwent a detailed clinical evaluation, which included an maximal CPET (and exercise ECG). ${ }^{25}$ These findings are additionally supported by the facts that the mean value of the maximum HR during CPET was about $98 \%$ of the predicted - 210 minus $(0.65 \mathrm{X}$ age), ${ }^{26}$ and a maximum oxygen uptake that was considerably higher than expected for their age (Table 4).

The evaluation of certain anthropometric measurements is practical, fast and almost costless. Our results show that, using simple measurements for BMI, waist circumference, somatotype and the sum of skinfolds, we were able to prematurely identify healthy men who already tend to have a lower CVT, a clinically potential useful information, since a reduction in the CVT indicates an increased risk for cardiovascular morbidity ${ }^{7}$ and mortality. ${ }^{6}$ From a practical point-of-view, a preliminary clinical approach, it would be to consider those subjects in the unfavorable extreme quintile as more prone to have a relatively lower vagal tone and then the ones that most likely will benefit of an intervention, such as lifestyle management (Table 3).

Our results are in agreement with other studies that have found a lower CVT in individuals who present criteria for excess of body fat. ${ }^{13,14}$ Some groups have reported that obesity and changes in weight can affect cardiovascular autonomic modulation. ${ }^{11-14,27}$ For instance, Chen et al..$^{13}$ found an association between waist circumference and a reduced CVT, while the same was not observed for BMI. These results may suggest that the fat distribution maybe more important for the reduction of CVT than overall or total body fat. It is important to note that, in this study, the authors used a sample consisting of two groups of individuals with and without obesity and of both genders. In our study, while the CVI values of extreme quintiles for BMI, waist circumference and the total sum of skinfolds were significantly different, indicating that healthy men that are not obese already present a trend for a lower CVT, the fat distribution appeared to not be so relevant, at least by PCI criteria. Indeed, the relationship of the PCI with the CVI is probably best represented by an inverted $\mathrm{U}$, with the $1^{\text {st }}$ and $5^{\text {th }}$ quintiles of the PCI presenting a lower mean CVI. 
The waist circumference, which is considered an important indicator of regional or local obesity and is associated with central fat, ${ }^{2,28}$ was the AC that presented the slightly higher correlation coefficient with the CVI ( $\mathrm{r}=-0.30$, $\mathrm{p}=<0.001$ ) (Table 3) and was also the $\mathrm{AC}$ with the greatest difference between the means of extreme quintiles for the CVI (first -1.77 vs. fifth $-1.46, \mathrm{p}<0.001$ ). These results, even in non-obese healthy subjects, are very similar to those obtained in other studies that involved the measurement of waist circumference in individuals with decreased CVI. ${ }^{4,5,13}$ These results are relevant since abdominal obesity is considered a risk factor for the development of $\mathrm{CVD}^{4,29}$ and an anthropometric variable associated with CVD. ${ }^{5,28}$ This information is very practical in terms of its availability and its simplicity of implementation in the practice of health professionals.

Another contribution of our study is the somatotype analysis. When considering the details of body composition, which include distinguishing between the effects of fat and skeletal muscle on body weight, somatotype analysis may eliminate potential errors caused by the crude use of body weight in relation to height, as is done when determining the BMI. ${ }^{30}$ This is particularly relevant when we note that increases in mesomorphy (based on limb girths subtracted from skinfolds, and measurements of bone diameters related to body height) are not related to CVI. Nevertheless, perhaps by either considering a much less controlled sample in terms of inclusion criteria or due to the use of previous somatotype classification systems such as Sheldon rather than Heath-Cater, these findings did not corroborate previous information available from years 1950 to 1960 gathered in an old review paper. ${ }^{31}$ Our results demonstrate that healthy individuals with a more endomorphic somatotype have a lower CVT, which may suggest a greater predisposition for the development of $\mathrm{CVD}^{31}$ and for mortality ${ }^{32}$ and, as found by Olson et al. ${ }^{32}$, in athletes and non-athletes, have lower life expectancy as compared to individuals with a predominantly mesomorphic and/or ectomorphic somatotype.
A possible limitation of our study was that we did not height-adjust the $\mathrm{AC}$ of our subjects. While this issue has been shown to be potentially relevant, according proportionality criteria, ${ }^{33}$ preliminary analysis applying these procedures were not effective in our sample, most likely because it is a very homogeneous sample with a mean height around $1.76 \mathrm{~m}$. However, it is possible that the correction of proportionality become appropriated when using a less homogeneous sample with a height distribution different from the one used in our study. For instance, for individuals that substantially differ in body height, there is a trend to also present large differences for other $\mathrm{AC}$ (e.g. waist circumference), and then, a height correction should be appropriate. Another limitation is the lack of scientific data in our study that could explain the possible physiopathological mechanisms involved in the reduction in the CVT in non-obese healthy men.

Future studies are necessary to confirm whether the lower CVT values observed in healthy men with UAC would represent an increased rate of cardiovascular events or, if this trend for a CVT reduction could be reversed with the correction of UAC by exercise and/or by a hypocaloric diet.

In conclusion, as more attention is being dedicated to the prevention and determination of long-term prognosis, the findings of this study contribute by indicate that even in healthy adult men with adequate levels of aerobic condition, it is already possible to identify those with a tendency to present lower CVT values through the use of easily obtained anthropometric measurements. In a preventive scenario, these findings may contribute to the early identification of healthy individuals in need for a greater emphasis and more aggressive regulation of cardiovascular risk factors.

\section{GRANT SUPPORT}

Authors were supported by the Brazilian National Council for Scientific and Technological Development CNPq.

\section{REFERENCES}

1. World Health Organization - Obesite and overweight. 2003. (Accessed April, 2009, at http://www.who.int/mediacentre/factsheets/fs311/en/.)

2. Balkau B, Deanfield JE, Despres JP, Bassand JP, Fox KA, Smith SC, Jr., et al. International Day for the Evaluation of Abdominal Obesity (IDEA): a study of waist circumference, cardiovascular disease, and diabetes mellitus in 168,000 primary care patients in 63 countries. Circulation. 2007;116:1942-51.

3. Gutin B, Johnson MH, Humphries MC, Hatfield-Laube JL, Kapuku GK, Allison JD, et al. Relationship of visceral adiposity to cardiovascular disease risk factors in black and white teens. Obesity (Silver Spring). 2007;15:1029-35.
4. Ricciardi R, Metter EJ, Cavanaugh EW, Ghambaryan A, Talbot LA Predicting cardiovascular risk using measures of regional and total body fat. Appl Nurs Res. 2009;22:2-9.

5. Pischon T, Boeing H, Hoffmann K, Bergmann M, Schulze MB, Overvad $\mathrm{K}$, et al. General and abdominal adiposity and risk of death in Europe. N Engl J Med. 2008;359:2105-20.

6. Lauer MS. Autonomic function and prognosis. Cleve Clin J Med 2009;76 Suppl 2:S18-22. 
7. Wichi RB, De Angelis K, Jones L, Irigoyen MC. A brief review of chronic exercise intervention to prevent autonomic nervous system changes during the aging process. Clinics. 2009;64:253-8.

8. Thayer JF, Lane RD. The role of vagal function in the risk for cardiovascular disease and mortality. Biol Psychol. 2007;74:224-42.

9. La Rovere MT, Bigger JT Jr., Marcus FI, Mortara A, Schwartz PJ. Baroreflex sensitivity and heart-rate variability in prediction of total cardiac mortality after myocardial infarction. ATRAMI (Autonomic Tone and Reflexes After Myocardial Infarction) Investigators. Lancet 1998;351(9101):478-84

10. Beske SD, Alvarez GE, Ballard TP, Davy KP. Reduced cardiovagal baroreflex gain in visceral obesity: implications for the metabolic syndrome. Am J Physiol Heart Circ Physiol. 2002;282:H630-5.

11. Laederach-Hofmann K, Mussgay L, Ruddel H. Autonomic cardiovascular regulation in obesity. J Endocrinol. 2000;164:59-66.

12. Piestrzeniewicz K, Luczak K, Lelonek M, Wranicz JK, Goch JH. Obesity and heart rate variability in men with myocardial infarction. Cardiol J. 2008;15:43-9.

13. Chen GY, Hsiao TJ, Lo HM, Kuo CD. Abdominal obesity is associated with autonomic nervous derangement in healthy Asian obese subjects. Clin Nutr. 2008;27:212-7.

14. Freeman R, Weiss ST, Roberts M, Zbikowski SM, Sparrow D. The relationship between heart rate variability and measures of body habitus. Clin Auton Res. 1995;5:261-6.

15. Alvarez GE, Davy BM, Ballard TP, Beske SD, Davy KP. Weight loss increases cardiovagal baroreflex function in obese young and older men. Am J Physiol Endocrinol Metab. 2005;289:E665-9.

16. Arone LJ, Mackintosh R, Rosenbaum M, Leibel RL, Hirsch J. Autonomic nervous system activity in weight gain and weight loss. Am J Physiol. 1995;269(1 Pt 2):R222-5.

17. Araújo CGS, Nobrega AC, Castro CL. Heart rate responses to deep breathing and 4-seconds of exercise before and after pharmacological blockade with atropine and propranolol. Clin Auton Res. 1992;2:35-40.

18. Ricardo DR, Almeida MB, Franklin BA, Araújo CGS. Initial and final exercise heart rate transients: influence of gender, aerobic fitness, and clinical status. Chest. 2005;127:318-27.

19. Norton K, T. O. Anthropometrica. Marrickville: Southwood Press; 1996.

20. Gomes PSC, Araújo CGS. Metodologia do somatotipo antropométrico de Heath-Carter. Caderno ARTUS de Medicina Desportiva. 1978;1:11-20.
21. Heath BH, Carter JE. A modified somatotype method. Am J Phys Anthropol. 1967;27:57-74.

22. Ross WD, Drinkwater DT, Bailey DA, Marshall GR, Leahy RM. Kinanthropometry: traditions and new perspectives. In: Ostyn M, Beunen G, Simons J, eds. Kinanthropometry II. Baltimore: University Park Press; 1980:3-26.

23. Buchfuhrer MJ, Hansen JE, Robinson TE, Sue DY, Wasserman K, Whipp BJ. Optimizing the exercise protocol for cardiopulmonary assessment. J Appl Physiol. 1983;55:1558-64

24. Myers J, Buchanan N, Smith D, Neutel J, Bowes E, Walsh D, et al Individualized ramp treadmill. Observations on a new protocol. Chest 1992;101(5 Suppl):236S-41S.

25. Araújo CGS, Pinto VLM. Maximal heart rate in exercise tests on treadmill an in a cycloergometer of lower limbs. Arq Bras Cardiol. $2005 ; 85: 45-50$

26. Lange-Anderson K, Shepard R, Denolin H, Varnauskas E, Masironi R Fundamentals of exercise testing. Geneva, Switzerland: World Health Organization; 1971

27. Skrapari I, Tentolouris N, Perrea D, Bakoyiannis C, Papazafiropoulou A, Katsilambros N. Baroreflex sensitivity in obesity: relationship with cardiac autonomic nervous system activity. Obesity. 2007;15:1685-93.

28. Dhaliwal SS, Welborn TA. Central obesity and multivariable cardiovascular risk as assessed by the Framingham prediction scores. Am J Cardiol. 2009;103:1403-7.

29. Chen L, Peeters A, Magliano DJ, Shaw JE, Welborn TA, Wolfe R, et al. Anthropometric measures and absolute cardiovascular risk estimates in the Australian Diabetes, Obesity and Lifestyle (AusDiab) Study. Eur J Cardiovasc Prev Rehabil. 2007;14:740-5.

30. Ricardo DR, Araújo CGS. Body mass index: a scientific evidence-based inquiry. Arq Bras Cardiol. 2002;79:61-78.

31. Williams S, Jones E, Bell W, Davies't B, Bourne M. Body habitus and coronary heart disease in men. Eur Heart J. 1997;18:376-93.

32. Olson H, Sprague H, Van Huss W. Somatotype and longevity of former University athletes and nonathletes. Res Q. 1990;61:1-6.

33. Ross WD, Wilson NCA. Stratagem for proportional growth assessment. Acta Paediatrica Belgica. 1974;28(Suppl):169-82. 
